\title{
SUPPRESSION OF SPURIOUS FREQUENCIES IN SCATTERING PROBLEMS BY MEANS OF BOUNDARY ALGEBRAIC AND COMBINED FIELD EQUATIONS
}

\author{
J. POBLET-PUIG, V.YU. VALYAEV AND A.V. SHANIN \\ Communicated by Paul Martin
}

\begin{abstract}
A numerical technique for solving scattering problems is presented. It is based on a boundary integral equation idea, so the unknowns are localized on the contour (in $2 \mathrm{D}$ case) or the surface (in 3D case) of the scattering object. Two major difficulties of traditional boundary integral methods (the appearance of spurious resonances and the necessity to perform numerical integration of singular functions) are overcome by studying the problem in an approximate discrete formulation from the very beginning. The space is filled by cubic blocks, and the shape of the scatterer is formed by a set of blocks removed from the space. Thus, the formulation of the problem is discrete, and the continuous Green's function is substituted by a discrete mesh Green's function. An analogue of combined field boundary integral equation (CFIE) is developed for this formulation.
\end{abstract}

1. Introduction. Scattering of waves by obstacles is a physical phenomenon emerging in several fields such as acoustics, electromagnetics, optics, seismology or marine engineering. In many cases, when time-harmonic dependence is assumed, the problem is governed by the Helmholtz equation which has to be solved in an infinite domain $\Omega^{\prime}$ :

$$
\begin{array}{ll}
\triangle u(\boldsymbol{x})+\mathcal{K}^{2} u(\boldsymbol{x})=f(\boldsymbol{x}) & \text { in } \Omega^{\prime}, \\
\frac{\partial u(\boldsymbol{x})}{\partial n}=0 & \text { on } \partial \Omega^{\prime},
\end{array}
$$

2010 AMS Mathematics subject classification. Primary 35P25, 65N80, 65R20.

This work has been supported by Russian government grant 11.G34.31.0066, Scientific school grant 2631.2012.2 and the Russian Foundation for Basic Research grant 12-02-00114.

Received by the editors on June 15, 2014, and in revised form on October 17, 2014. 
Here, $\partial \Omega^{\prime}$ is the boundary of the scatterer and $\mathcal{K}$ is the wavenumber. The sources $f(\boldsymbol{x})$ are non-zero in a finite domain in the space.

An important part of problem formulation is the radiation condition preventing waves coming from infinity. Here, we prefer to formulate this condition in the form of the limiting absorption principle. Namely, in the case $\operatorname{Im}[\mathcal{K}]>0$, we look for the solution decaying exponentially as $|x| \rightarrow \infty$. In the case $\operatorname{Im}[\mathcal{K}]=0$, the solution is looked for in the sense of the limit $\operatorname{Im}[\mathcal{K}] \rightarrow 0$.

A considerable variety of numerical techniques have been developed to model wave problems and deal with infinite domains in different physical formulations [52]. Domain discretization techniques, such as the finite element method (FEM) or finite differences (FD), require a domain truncation and some non-reflecting boundary condition to be imposed. See, for example, the technique of perfectly matching layers (PML) [54] or the use of infinite elements [17], among others. On the contrary, in the boundary integral formulations, only the boundary of the scatterer is discretized and the unknowns belong only to this boundary. In these methods, radiation condition is satisfied in a natural way.

However, boundary formulations also have some drawbacks. First of all, the related linear systems of equations depend on the frequency through the Green's function of the problem. This implies that matrices must be calculated for each frequency (typically, engineering applications require a lot of frequencies). Formally, matrices for the boundary element method (BEM) have smaller dimension than that of the FEM, but the matrices for the BEM are densely populated (as opposed to the FEM, where the matrices are sparse). This aspect can balance the cost of the solution of linear systems written for the BEM and the FEM [21].

In order to calculate the matrices for one of the BEM, singular integrals have to be precisely evaluated by means of specific numerical techniques. The degree of singularity can vary depending on several aspects, such as the relative position between the source and the field points (or the element calculated) and the type of kernel functions. Some techniques have been developed in order to perform proper integrations: element subdivision, adaptive Gauss-Legendre integration, variable transforms or semi-analytical integration based on series ex- 
pansions, among others $[39,36,49,23,19]$.

Boundaries with corners or angular shapes, which are frequent in engineering, can also cause problems for the BEM. Several mathematical studies $[41,35]$ show that the theoretical basis of the BEM can fail in the presence of irregular boundaries, since the linear systems of equations become ill-conditioned or nearly singular. From the practical point of view, some of the problems are caused by the non-unique normal derivative at the corner. A possibility of handling this duplicity of values is to double nodes in the corners [20]. Then, additional equations that avoid repeated information are obtained by using alternative collocation points. A review of other techniques or improved integral equations can be found in $[42, \mathbf{1 3}, \mathbf{2}]$. Moreover, the presence of scatterers with corners or sharp edges often leads to a solution with singularities. In these cases, standard interpolations cannot accurately reproduce the solution; numerical errors are larger than expected and convergence rates are reduced [22].

Equations corresponding to the simplest BEM formulations are known to be ill-posed for the frequencies close to the eigenfrequencies of the scatterer. There exist a duality between an exterior problem and the equivalent interior problem which is responsible for the spurious frequencies [25]. This is only a numerical or theoretical pathology with no physical correspondence. It means that, in real life or in cases where an analytical solution of the problem is available, no evidence of this phenomenon is observed. In order to overcome this difficulty several options have been proposed. On the one hand, the combined field integral equations $[37,8]$ (CFIE) where, instead of solving the simple boundary integral equation, a combination of this equation plus some variation (e.g., normal derivative) of the original one multiplied by a complex constant is solved. The main drawback of these techniques is that hypersingular integrals (singularities of $o\left(1 / r^{2}\right)$ type for twodimensional or $o\left(1 / r^{3}\right)$ type for three-dimensional problems) can appear due to the secondary derivation of the Green's functions with respect to the surface normal. Efficient methods to compute these integrals have been proposed [14]. Improvements and modifications of the idea exist, see for example, [16] where some interior points (not only nodes on the boundary) are considered. However, the boundary integrals with strong singularities remain a common aspect of all CFIE-based approaches. 
The combined Helmholtz integral equation formulation (CHIEF) method [46] uses a standard boundary integral equation plus some additional equations generated by placing collocation points in the interior of the scatterer. These additional equations specify that the variable inside the scatterer must be null. These new equations are redundant for frequencies that do not coincide with the scatterer eigenfrequencies, but they are necessary at the spurious resonance frequencies. The main drawback of the method is that it can fail if the interior points coincide with the nodes (zones of null value) of the scatterer eigenfunction. These nodes are not known a priori; thus, the probability of failure of the method is quite heuristic. A comparison between CHIEF and CFIE methods can be found in [1], and a comparison with other techniques can be found in $[4,12]$.

The goal of our work is to present a numerical method that possesses the advantages of a boundary integral formulation but that avoids the difficulties caused by singular integrals. The method is also free of spurious resonance frequencies due to using a discrete version of the CFIE formulation. In fact, the main contribution of the technique is to avoid the hypersingular integrals of CFIE formulation by means of a discrete formulation of the problem that uses the corresponding discrete Green's function.

The formulation is particularized for the Helmholtz equation. An operator on a square grid is considered (e.g., based on finite differences). Thus, geometries must be approximated by the closest brick-shaped equivalent description. The method is conceived for engineering applications where it is more important to obtain a fast and correct description of the solution rather than to obtain a very precise approximation. The effort is focused here in the generalization of the method to several operators. Two aspects must be handled: the truncation of the operator and the definition of specific discrete Green's functions. The efficiency and robustness of the discrete CFIE formulation is studied by means of several numerical examples.

Discrete Green's functions were also considered to study diffraction problems in exterior domains $[\mathbf{3}, \mathbf{6}]$. This idea of first doing the discretization and afterwards reducing the problem to the boundary has been used in order to deal with arbitrary shaped interior domains and lattices with periodic irregularities $[\mathbf{3 0}, \mathbf{1 8}]$. The analysis is mainly focused in the study of spectral properties of Laplace operator. 
Another important method that can be used to reduce the problem to the boundary is the method of difference potentials $[44,45,53]$. It is based on a different boundary integral equation that deals with single and double layer potentials and allows for a discontinuity of the variable in the boundary of the studied domain. This approach is not prone to any adverse effects related to the resonances of the complementary domains. Moreover, it is quite general to allow for several discrete versions (i.e., based on finite differences [53]). An adequate choice of the potentials makes the method more flexible in the sense that Green's functions are not strictly required. Several descriptions of the boundary geometry can also be considered. However, they can increase the complexity of the implementation (modify the coordinate system or perform a numerical description of the boundary and normal derivatives). This method has been applied in practice to the active control of noise by acting only on the boundary of the domain [28] and also to develop high-order methods and make an accurate treatment of the problem geometry[32].

The Helmholtz equation in exterior domains has already been solved by means of algebraic equations reduced to the boundary in [51]. The main goal of this work is to show how this technique avoids the singular integrals that are often involved in the BEM.

An outline of the paper is as follows. The theoretical basis and main ideas of the method are presented in Sections 2 and 3. Section 4 deals with implementation details, including the evaluation of discrete Green's functions, the description of the scatterer and the consideration of different incident fields. Some details related to Green's functions are repeated for convenience. Also, the first part before CFIE formulation could be obtained with the procedures proposed in $[\mathbf{3 0}, \mathbf{1 8}]$. However, the equations are detailed from the beginning for coherence with CFIE formulation exposed latter. In the numerical examples of Section 5, a very simple one-dimensional case with almost analytical formulation is studied. The properties of the technique are shown by means of two-dimensional examples. The numerical results are compared with analytical results (where available) and results obtained by means of other numerical techniques. Future development is proposed in Section 6. 


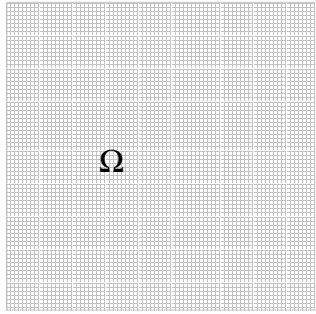

(a)

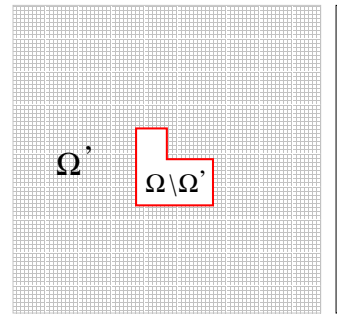

(b)

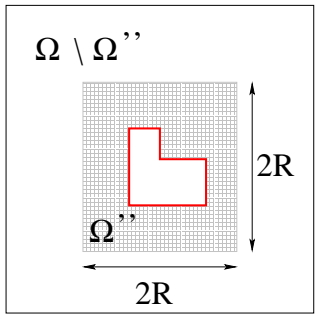

(c)

FIGURE 1. Domains involved in the formulation: (a) unbounded without scatterer $\Omega$; (b) unbounded but truncated with boundary $\partial \Omega^{\prime}$ around the scatterer; (c) $\Omega^{\prime \prime}$ finite truncation of $\Omega^{\prime}$.

2. Problem formulation. Consider a cubic (three-dimensional) or rectangular (two-dimensional) grid of the problem domain $\Omega^{\prime} . \Omega^{\prime}$ is a part of the whole two- or three-dimensional space $\Omega$. The nodes are denoted by the index $j$. We also define a mesh $\Omega^{\prime \prime}$ which is a finite truncation of $\Omega^{\prime}$. As a truncation, we take for clarity a large square or cube of side $2 R$ with the origin of the coordinate system in the center. We assume that the diameter of the scatterer is much smaller than $R$ and that the origin belongs to the scatterer, i.e., to $\Omega \backslash \Omega^{\prime}$.

We call two nodes the neighbors if the nodes belong to the same finite element (elementary square or cube) of the full mesh $\Omega$.

Define the boundary of the scatterer $\partial \Omega^{\prime}$ as the set of all neighbors of the nodes belonging to $\Omega \backslash \Omega^{\prime}$ in $\Omega^{\prime}$. Nodes of $\Omega^{\prime} \backslash \partial \Omega^{\prime}$ will be called the internal nodes of $\Omega^{\prime}$. Define also the exterior boundary of $\partial \Omega^{\prime \prime}$ as the set of neighbors of $\Omega^{\prime} \backslash \Omega^{\prime \prime}$.

Define linear operators $L, L^{\prime}$ and $L^{\prime \prime}$ on the meshes $\Omega, \Omega^{\prime}$ and $\Omega^{\prime \prime}$, respectively. The definition is as follows:

$$
\begin{aligned}
L[u]_{j} & \equiv \sum_{q \in \Omega} \beta_{j, q} u_{q}, \quad j \in \Omega, \\
L^{\prime}[u]_{j} & \equiv \sum_{q \in \Omega^{\prime}} \beta_{j, q}^{\prime} u_{q}, \quad j \in \Omega^{\prime}, \\
L^{\prime \prime}[u]_{j} & \equiv \sum_{q \in \Omega^{\prime \prime}} \beta_{j, q}^{\prime \prime} u_{q}, \quad j \in \Omega^{\prime \prime},
\end{aligned}
$$


Matrices of the coefficients $\beta_{j, q}, \beta_{j, q}^{\prime}, \beta_{j, q}^{\prime \prime}$ have the following properties:

(i) the elements $\beta_{j, q}, \beta_{j, q}^{\prime}, \beta_{j, q}^{\prime \prime}$ are equal to zero if $q$ and $j$ are not neighbors;

(ii) matrices are symmetrical, i.e.,

$$
\beta_{j, q}=\beta_{q, j}, \quad \beta_{j, q}^{\prime}=\beta_{q, j}^{\prime}, \quad \beta_{j, q}^{\prime \prime}=\beta_{q, j}^{\prime \prime}
$$

for all admissible $j$ and $q$;

(iii) operator $L^{\prime}$ is a truncation of $L$, and operator $L^{\prime \prime}$ is a truncation of $L^{\prime}$ in the following specific sense:

$$
\beta_{j, q}^{\prime}=\beta_{j, q} \quad \text { if } j, q \in \Omega^{\prime}, \quad j \text { or } q \notin \partial \Omega^{\prime} .
$$

The condition means that $j$ or $q$ is one of the internal nodes of $\Omega^{\prime}$. Similarly,

$$
\beta_{j, q}^{\prime \prime}=\beta_{j, q}^{\prime} \quad \text { if } j, q \in \Omega^{\prime \prime}, \quad j \text { or } q \notin \partial \Omega^{\prime \prime} .
$$

According to the first property, all sums in (2.1), (2.2) and (2.3) are finite for each $j$.

We assume that all operators introduced above approximate the Helmholtz equation in corresponding areas. Let $L^{\prime}$ approximate Neumann boundary conditions on the boundary $\partial \Omega^{\prime}$. We do not specify the details of operator $L^{\prime \prime}$ on the boundary $\partial \Omega^{\prime \prime}$. The inhomogeneous equation

$$
L^{\prime}[u]_{j}=f_{j} \text { for all } j \in \Omega^{\prime},
$$

approximates equation 1.1 with Neumann condition on the boundary of the scatterer. When solving equation2.7, we assume that $\operatorname{Im}[\mathcal{K}]>0$, that the sources $f_{j}$ are localized in a finite area, and we look for the solution decaying exponentially at infinity.

We assume also that matrix $\beta$ is homogeneous with respect to translations along the coordinate lines. This property will enable us to use Fourier transform to compute Green's function of $L$.

Examples of operator $L$ are provided in subsection 4.1. The nodes are called edge-adjacent if they are adjacent along one of the coordinate lines, $h$ is the size of the grid. 
3. Discrete approximations of integral equations. Let $G_{j}^{m}$ be the discrete Green's function of the mesh $\Omega$, i.e., a solution of equation

$$
L\left[G^{m}\right]_{j}=\delta_{j, m},
$$

where $\delta_{j, m}$ is the Kronecker delta (unit force is placed at node $m$ ). According to the limiting absorption principle for each complex value of $\mathcal{K}$ we choose the Green's function that decays exponentially at infinity.

Consider operator $L^{\prime \prime}$ defined on a finite mesh. The following relation follows from (2.4). For any functions $u_{j}$ and $w_{j}$ defined on $\Omega^{\prime}$,

$$
\sum_{j \in \Omega^{\prime \prime}} L^{\prime \prime}[u]_{j} w_{j}=\sum_{j \in \Omega^{\prime \prime}} L^{\prime \prime}[w]_{j} u_{j} .
$$

Let $L^{\prime}[u]_{j}$ and $L^{\prime}[w]_{j}$ be non-zero in a finite area of the mesh, and let both functions obey the radiation condition formulated above. The operator $L^{\prime \prime}$ differs from $L^{\prime}$ on $\Omega^{\prime \prime}$ only on the boundary $\partial \Omega^{\prime \prime}$. According to the limiting absorption principle, this difference decays exponentially as $R \rightarrow \infty$. Thus, we can take this limit and replace $\Omega^{\prime \prime}$ by $\Omega^{\prime}$ :

$$
\sum_{j \in \Omega^{\prime}} L^{\prime}[u]_{j} w_{j}=\sum_{j \in \Omega^{\prime}} L^{\prime}[w]_{j} u_{j}
$$

Taking in (3.3) $\Omega^{\prime}$ as $\Omega, G_{j}^{m}$ as $u_{j}$, and $G_{j}^{n}$ as $w_{j}$ one can prove that

$$
G_{m}^{n}=G_{n}^{m}
$$

Restrict function $G_{j}^{m}$ to the mesh $\Omega^{\prime}$. Formally, the restriction is a different function, but we denote it below with the same symbol $G_{j}^{m}$. Apply operator $L^{\prime}$ to the restriction $G_{j}^{m}$. Obviously, equation (3.1) will not be valid for operator $L^{\prime}$, since the operators $L^{\prime}$ and $L$ are different on $\partial \Omega^{\prime}$. Instead, for $m \in \Omega^{\prime}$, define the values $b_{j}^{m}$ by the following relation:

$$
L^{\prime}\left[G^{m}\right]_{j}=\delta_{j, m}+b_{j}^{m} .
$$

These values play the role of boundary residue of operator $L^{\prime}$. The values $b_{j}^{m}$ are not equal to 0 only if $j \in \partial \Omega^{\prime}$. 
Taking $w_{j}=G_{j}^{m}$ in equation (3.3), we derive the following "boundarydifference representation" for the field:

$$
\begin{aligned}
u_{m} & =\sum_{j \in \Omega^{\prime}}\left(f_{j} G_{j}^{m}-u_{j} b_{j}^{m}\right) \\
& =\sum_{j \in \Omega^{\prime}} f_{j} G_{j}^{m}-\sum_{j \in \partial \Omega^{\prime}} u_{j} b_{j}^{m} \quad \text { for all } m \in \Omega^{\prime} .
\end{aligned}
$$

We are particularly interested in the case $m \in \partial \Omega^{\prime}$ because then a kind of "boundary integral equation" (BIE) is obtained. The values of $u_{j}$, $j \in \partial \Omega^{\prime}$ are the unknowns of this system.

Equation (3.6) plays the role of boundary integral equation in direct Kirchhoff formulation. Theoretically, it can be used to find the boundary values of $u$, but in practice it is prone to spurious resonances. Our next step is to correct (3.6) by adding some other equation. Ideologically, this step mimics the CFIE approach to boundary integral equations.

Apply operator $L^{\prime}$ to both sides of (3.6), and take into account that $f_{m}=L^{\prime}[u]_{m}$ :

$$
f_{m}=\sum_{j, n \in \Omega^{\prime}} f_{j} G_{j}^{n} \beta_{m, n}^{\prime}-\sum_{j \in \partial \Omega^{\prime}} u_{j} \sum_{n \in \Omega^{\prime}} b_{j}^{n} \beta_{m, n}^{\prime} .
$$

This equation is valid for any $m \in \Omega^{\prime}$, but we will be interested only in the case $m \in \partial \Omega^{\prime}$. According to the general scheme of the CFIE method (see [8]), make a linear combination of (3.6) and (3.7).

$$
\begin{aligned}
u_{m}+\nu f_{m}= & \sum_{j, n \in \Omega^{\prime}} f_{j}\left(\delta_{m, n}+\nu \beta_{m, n}^{\prime}\right) G_{j}^{n} \\
& -\sum_{j \in \partial \Omega^{\prime}} u_{j} \sum_{n \in \Omega^{\prime}}\left(\delta_{m, n}+\nu \beta_{m, n}^{\prime}\right) b_{j}^{n} .
\end{aligned}
$$

$\nu$ is the combination parameter that must be complex. Taken for $m \in \partial \Omega^{\prime},(3.8)$ is a linear system for finding the boundary values of $u_{m}$. Note that, although summation over $n$ in the second term is formally held over the whole mesh $\Omega^{\prime}$, in practice it is held over the finite set of neighbors of $\partial \Omega^{\prime}$.

Equation (3.8) corresponds to the radiation problem (2.7). Usually, a diffraction problem should be solved, i.e., the incident field $u^{\text {in }}$ is given, such that it obeys equation $L\left[u^{\mathrm{in}}\right]_{j}=0$ on $\Omega$. It is necessary to 
find the scattered field $u_{j}$ such that the total field $u^{\text {tot }}=u^{\text {in }}+u$ obeys the homogeneous equation $L^{\prime}\left[u^{\text {tot }}\right]_{j}=0$ everywhere on $\Omega^{\prime}$. In this case, we can again use equation (3.8) taking

$$
f_{j}=-L^{\prime}\left[u^{\mathrm{in}}\right]=-\sum_{q \in \Omega^{\prime}} \beta_{j, q}^{\prime} u_{q}^{\mathrm{in}} .
$$

Note that (3.9) cannot be substituted directly into (3.8) since, generally, $u^{\text {in }}$ does not obey the radiation condition (i.e., it is not exponentially decaying for $\operatorname{Im}[\mathcal{K}]>0$ ) and thus it cannot be used in (3.3) as one of the functions. To compute the force term using equation (3.9) is completely equivalent to imposing the null normal derivative of the total field $u^{\text {tot }}$ around the scatterer.

Equation (3.8) can be rewritten in a form more suitable for computations, namely, as

$$
\sum_{j \in \partial \Omega^{\prime}} M_{m, j} u_{j}=\sum_{j \in \Omega^{\prime}} F_{m, j} f_{j}, \quad m \in \partial \Omega^{\prime},
$$

where

$$
\begin{gathered}
M_{j, q}=\nu \sum_{n, q \in \Omega^{\prime}} \beta_{m, n}^{\prime} G_{q}^{n} \beta_{q, j}^{\prime}+\sum_{q \in \Omega^{\prime}} G_{q}^{m} \beta_{q, j}^{\prime}-\nu \beta_{m, j}^{\prime}, \quad j, q \in \partial \Omega^{\prime} \\
F_{m, j}=\nu \sum_{q \in \Omega^{\prime}} \beta_{m, q}^{\prime} G_{j}^{q}+G_{j}^{m}-\nu \delta_{m, j}, \quad m \in \partial \Omega^{\prime}, j \in \Omega^{\prime} .
\end{gathered}
$$

Equation (3.10) will be solved below with respect to $u_{j}$. In terms of the acoustical problem, this means finding the surface pressures on the surface of the scatterer. Then representation (3.6) can be used for finding the pressures everywhere in $\Omega^{\prime}$.

Values of $\nu$ with non-null imaginary part ensure the uniqueness of equation (3.8) for all wavenumbers. The proof exposed in [8] is adapted in $[\mathbf{3 8}]$ for the discrete problem.

\section{Implementation details.}

4.1. Discrete operator and the description of the scatterer. The more general framework of Section 3 is particularized now for several operators. Each option leads to a different set of coefficients 
$\beta^{\prime}$. The two-dimensional field operator can be expressed as

$$
L[u]_{j}=A_{0} u_{j_{1}, j_{2}}+A_{s} \bar{u}_{j_{1}, j_{2}}+A_{c} \widehat{u}_{j_{1}, j_{2}}
$$

with

$$
\bar{u}_{j_{1}, j_{2}}=u_{j_{1}+1, j_{2}}+u_{j_{1}-1, j_{2}}+u_{j_{1}, j_{2}+1}+u_{j_{1}, j_{2}-1}
$$

and

$$
\widehat{u}_{j_{1}, j_{2}}=u_{j_{1}+1, j_{2}+1}+u_{j_{1}-1, j_{2}+1}+u_{j_{1}-1, j_{2}-1}+u_{j_{1}+1, j_{2}-1} .
$$

The values of coefficients $A_{0}, A_{s}, A_{c}$ depend on the discretization technique. Some examples can be found in Table 1.

TABLE 1. Coefficients of the two-dimensional operator based on: Finite differences point-wise representation (FD), linear finite element method (FEM), linear finite element method with lumped mass matrix (FEM-LUMP).

\begin{tabular}{cccc} 
Method & $h^{2} A_{0}$ & $h^{2} A_{s}$ & $h^{2} A_{c}$ \\
\hline FD & $-4+(\mathcal{K} h)^{2}$ & 1 & 0 \\
FEM & $\frac{-8}{3}+\frac{4(\mathcal{K} h)^{2}}{9}$ & $\frac{1}{3}+\frac{(\mathcal{K} h)^{2}}{9}$ & $\frac{1}{3}+\frac{(\mathcal{K} h)^{2}}{36}$ \\
FEM-LUMP & $\frac{-8}{3}+(\mathcal{K} h)^{2}$ & $\frac{1}{3}$ & $\frac{1}{3}$ \\
\hline
\end{tabular}

An important aspect of the method is the definition of the truncated operator $L^{\prime}$. In this step, the geometry of the scatterer is described and the discrete normal derivative implicitly defined.

In all two-dimensional cases analyzed here (finite differences, finite element) the operator $L^{\prime}$ can be expressed in a unified framework similar to a standard finite element formulation

$$
\begin{aligned}
L^{\prime}[u]_{j} & =\sum_{i \in \Omega^{\prime}} \beta_{j, i}^{\prime} u_{i}=\frac{1}{h^{2}} \sum_{i \in \Omega^{\prime}}\left(-K_{j, i}+(\mathcal{K} h)^{2} \frac{M_{j, i}}{4}\right) u_{j} \\
& =\left\{\begin{array}{cc}
f\left(j_{1}, j_{2}, j_{3}\right) & \text { for FD } \\
\frac{1}{h^{2}}\left(\int_{\Omega^{\prime}} N_{i} f_{j} d \Omega-\int_{\partial \Omega^{\prime}} N_{i} \frac{\partial u}{\partial n} d \Gamma\right) & \text { for FEM. }
\end{array}\right.
\end{aligned}
$$

'FD' specifies that the boundary algebraic formulation is based on the finite difference equation. Then, operator $L$ is obtained by means of finite differences. In the 'FEM' case, the field operator $L$ is obtained by means of the weak form of the problem (as finite elements do). In that second case the force term includes a part related to the sources 
inside $\Omega^{\prime}$ and another related to imposed normal derivatives on $\partial \Omega^{\prime}$. $N_{i}$ is the FEM shape function in the reference element. This is an important advantage with respect to the formulation based on finite difference equation where the imposed velocities on $\partial \Omega^{\prime}$ are not so directly included.

The values of $K_{j, i}$ and $M_{j, i}$ depend on the discretization type. In the 'FEM' case they are the coefficients of the elemental stiffness and mass matrices in the reference element (see Figure 2 (b))

$$
K_{j i}=\iint_{-1}^{1} \nabla N_{i} \cdot \nabla N_{j} d \xi d \eta \quad M_{j i}=\iint_{-1}^{1} N_{i} N_{j} d \xi d \eta .
$$

In the 'FD' case, $K_{j i}$ and $M_{j i}$ values are chosen in order to reproduce the operator $L$ of equation (4.1) after assembling all the elements surrounding a node.

These elemental matrices have the standard structure

$$
\left[\begin{array}{llll}
a_{0} & a_{s} & a_{c} & a_{s} \\
a_{s} & a_{0} & a_{s} & a_{c} \\
a_{c} & a_{s} & a_{0} & a_{s} \\
a_{s} & a_{c} & a_{s} & a_{0}
\end{array}\right]
$$

with the coefficients defined in Table 2 .

TABLE 2. Coefficients of the two-dimensional local matrices $\mathbf{K}$ and $\mathbf{M}$.

\begin{tabular}{cccc|ccc} 
& \multicolumn{3}{c|}{$\mathbf{K}$} & & $\mathbf{M}$ \\
& FD & FEM & FEM lumped & FD & FEM & FEM lumped \\
\hline$a_{0}$ & 1 & $2 / 3$ & $2 / 3$ & $1 / 4$ & $1 / 9$ & $1 / 4$ \\
$a_{s}$ & $-1 / 2$ & $-1 / 6$ & $-1 / 6$ & 0 & $1 / 18$ & 0 \\
$a_{c}$ & 0 & $-1 / 3$ & $-1 / 3$ & 0 & $1 / 36$ & 0 \\
\hline
\end{tabular}

Operator $L^{\prime}$ can be obtained for arbitrary geometries in a procedure which is similar to the standard way of assembling elements in a finite element code. For each node in $\partial \Omega^{\prime}$ the existing square elements that contain this node must be assembled. According to the local numbering of nodes in Figure 2 (b) and the notation for the square elements surrounding a node in Figure 2 (a), it can be seen that a given node $j$ is in the local position $i$ of the square element $q_{i}$. Consequently, the row $i$ of the local matrix in equation (4.6) must be assembled. 
Consider as an example the node A in Figure 2 (c). In that node, square elements $q_{1}$ and $q_{2}$ exist, and the operator $L^{\prime}$ is constructed by assembling rows 1 and 2 of the elemental matrices $K$ and $M$ according to equation (4.4).

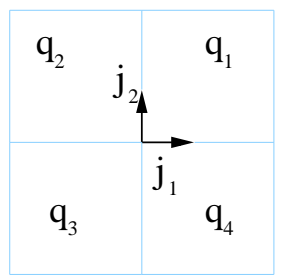

(a)

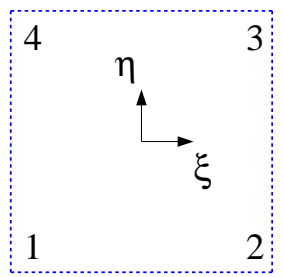

(b)

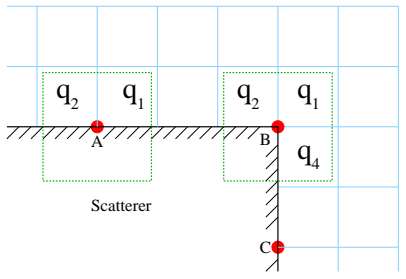

(c)

FIgURE 2. Two-dimensional description of the scatterer and creation of the operator $L^{\prime}$ on its boundary by means of an element-based concept: (a) Notation of the quadrants surrounding a node; (b) Local notation of nodes in the linear quadrilateral (square) finite element; (c) Examples of several situations of a node on the scatterer boundary: $\mathrm{A}$ and $\mathrm{C}$ are side nodes while $\mathrm{B}$ is a corner node.

With the coefficients in Table 2, coefficients of $L^{\prime}$ can be constructed a priori, and this procedure is done only once.

An example of application of this technique is given for the $2 \mathrm{D}$ case based on the finite difference operator. In Figure 3, a sample scatterer is demonstrated. One can see that the boundary nodes can be divided into 12 types. Elements of $L^{\prime}$ in the vicinity of each type of node are given in Table 3.

Alternative, high-order operators could be considered. The formulation in Sections 2 and 3 remains valid in these cases by only considering 


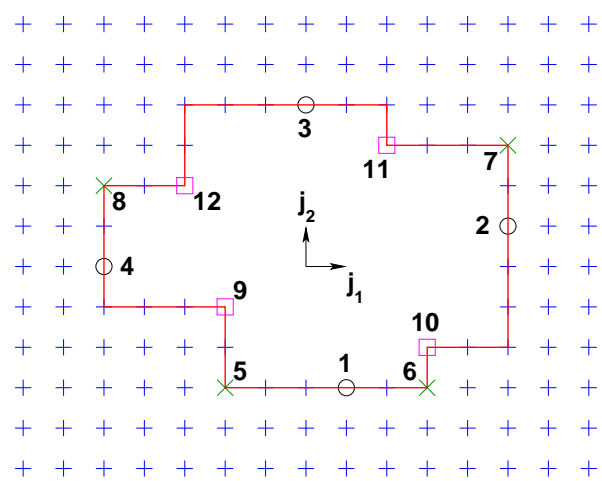

FIGURE 3. Sketch of the two-dimensional scatterer. The 12 node types in the boundary are identified.

TABLE 3. Weights of the finite difference operator in two-dimensional problems according to (2.7).

\begin{tabular}{c|ccccc}
$\begin{array}{c}\text { node } \\
\text { type }\end{array}$ & $\left(j_{1}-1, j_{2}\right)$ & $\left(j_{1}+1, j_{2}\right)$ & $\begin{array}{c}h^{2} \times \beta^{\prime} \\
\left(j_{1}, j_{2}-1\right)\end{array}$ & $\left(j_{1}, j_{2}+1\right)$ & $\left(j_{1}, j_{2}\right)$ \\
\hline 0 & 1 & 1 & 1 & 1 & $-4+(\mathcal{K} h)^{2}$ \\
1 & $1 / 2$ & $1 / 2$ & 1 & - & $-2+(\mathcal{K} h)^{2} / 2$ \\
2 & - & 1 & $1 / 2$ & $1 / 2$ & $-2+(\mathcal{K} h)^{2} / 2$ \\
3 & $1 / 2$ & $1 / 2$ & - & 1 & $-2+(\mathcal{K} h)^{2} / 2$ \\
4 & 1 & - & $1 / 2$ & $1 / 2$ & $-2+(\mathcal{K} h)^{2} / 2$ \\
5 & 1 & $1 / 2$ & 1 & $1 / 2$ & $-3+3(\mathcal{K} h)^{2} / 4$ \\
6 & $1 / 2$ & 1 & 1 & $1 / 2$ & $-3+3(\mathcal{K} h)^{2} / 4$ \\
7 & $1 / 2$ & 1 & $1 / 2$ & 1 & $-3+3(\mathcal{K} h)^{2} / 4$ \\
8 & 1 & $1 / 2$ & $1 / 2$ & 1 & $-3+3(\mathcal{K} h)^{2} / 4$ \\
9 & $1 / 2$ & - & $1 / 2$ & - & $-1+(\mathcal{K} h)^{2} / 4$ \\
10 & - & $1 / 2$ & $1 / 2$ & - & $-1+(\mathcal{K} h)^{2} / 4$ \\
11 & - & $1 / 2$ & - & $1 / 2$ & $-1+(\mathcal{K} h)^{2} / 4$ \\
12 & $1 / 2$ & - & - & $1 / 2$ & $-1+(\mathcal{K} h)^{2} / 4$ \\
\hline
\end{tabular}

a more general definition of $\beta$ coefficients because standard high-order finite difference schemes or finite elements often relate non-adjacent nodes. Also, discrete Green's functions can be evaluated numerically even if the formulas are a bit more complex. However, some difficulties arise from the implementation point of view. On the one hand, 
the unknowns are not only the nodes on the boundary of the scatterer but also some layer around it. For example, in the case of the crossshaped fourth order finite difference stencil that includes nine points, the method requires an additional layer of nodes around the boundary (coefficients $b_{j}^{m}$ become non-null there). On the other hand, and more importantly, it is much more difficult to impose boundary conditions and define a proper truncation of the operator $L$ when coefficients $\beta$ of non-adjacent nodes can be non-null.

A good alternative could be the compact high-order finite difference schemes proposed in $[48,34]$ and used in [31] with the difference potential method.

The use of a high-order operator in the proposed approach should be restricted to staircased geometries. Otherwise, the increase in accuracy caused by the better interpolation could be masked by a poor approximation of the problem geometry. Few studies have incorporated the geometry error in the error indicator [27]. However, in most of them (see, for example, for the FEM [40] or for the BEM [26]) the geometry error is estimated by means of the distance $d$ between the real boundary and its discrete approximation (i.e., in two-dimensional problems: a triangulation of the domain in the FEM, an approximation by means of straight segments in the linear BEM or a staircased geometry in the approach presented here).

Assuming that the exact geometry is a surface of constant curvature (radius of curvature $R$ ) in the BEM discretization of the boundary (straight elements of length $h$ ) shown in Figure 4 (a), this distance $d$ is

$$
d=R\left(1-\cos \left(\arcsin \left(\frac{h}{2 R}\right)\right)\right)
$$

and using Taylor series around $h=0$ we have

$$
d \simeq \frac{1}{8 R} h^{2}+o\left(h^{4}\right), \quad \frac{h}{R} \ll 1 .
$$

For the case of the staircased geometry with grid size $h$ as shown in Figure 4 (b), the distance between a node and the exact boundary can be expressed as

$$
d=R\left(1-\cos \left(\arcsin \left(\frac{\sqrt{2} h}{2 R}\right)\right)\right)+\frac{\sqrt{2} h}{2}
$$




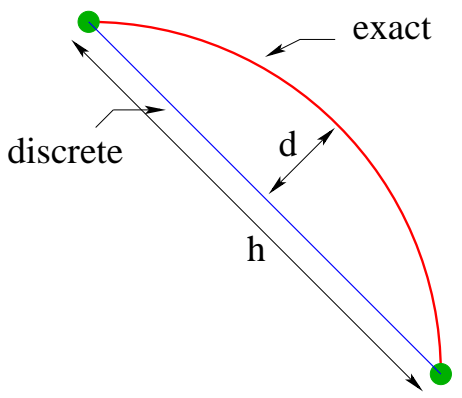

(a)

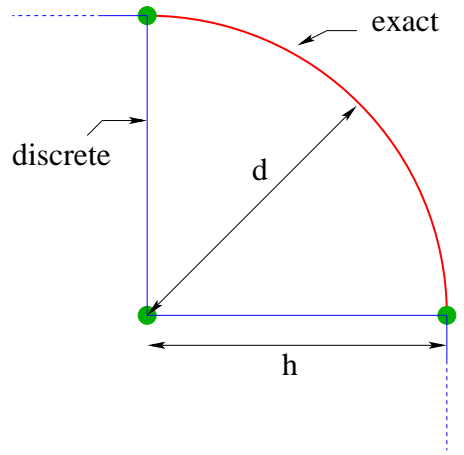

(b)

Figure 4. Distance $d$ between exact (red) and approximated (blue) geometries: (a) straight segments (BEM); (b) Staircased.

and again using the Taylor series it becomes

$$
d \simeq \frac{h}{\sqrt{2}}+\frac{h^{2}}{4 R}+o\left(h^{4}\right), \quad \frac{\sqrt{2} h}{R} \ll 1 .
$$

Comparing equation 4.8 and 4.10 it can be seen that the asymptotic dependence of $d$ on $h$ is of different order (order one in staircased geometry, which is worse). This has a direct consequence in the estimation of the geometric error which is based on a relationship of the type $d=C h^{n}$. The geometry approximation error of finite elements is proportional to $h^{3 / 2}$ ( $h$ is the element size) [40]. In the derivation to this error bound it is used that the distance between an smooth curved boundary and its triangulation is proportional to $h^{2}$, and the final result is not dependent on the order of polynomial interpolation used for the problem variable. For this reason, a high order method used in a curved geometry problem does not converge with proper rate corresponding to the high-order polynomial interpolation unless the geometry is described with the same accuracy. For example, finite elements using interpolation functions of order $p \geq 2$ and linear interpolation of the curved problem geometry do not converge according to the high-order $p$ of the polynomial but as linear elements. 
4.2. Boundary conditions. One of the main advantages of using an element approach is that boundary conditions are imposed naturally by means of the proper definition of operator $L^{\prime}$ of subsection 4.1. In fact, $L^{\prime}[u]_{m}$ is equivalent to the external normal derivative.

Consider as an example the 'FD' based option. In node $\mathrm{C}$ (with coordinates $j_{1}$ and $j_{2}$ ) of Figure 2 (c), the second order expression of the derivative is:

$$
\frac{d u}{d x}=\frac{u_{j_{1}+1, j_{2}}-u_{j_{1}-1, j_{2}}}{2 h}+o\left(h^{2}\right)=-\mathbf{n} \cdot \nabla u=-\frac{\partial u}{\partial n},
$$

the value of $u_{j_{1}-1, j_{2}}$ in the fictitious node from equation (4.11) can be isolated and replaced in equation (4.1) in order to obtain $L^{\prime}[u]_{C}$. This is a common procedure used in finite differences in order to impose Neumann boundary conditions. It is fully equivalent to the assembly procedure proposed here where the operator $L^{\prime}$ is obtained after assembling the elements $q_{1}$ and $q_{4}$ with node $\mathrm{C}$ in local positions 1 and 4 , respectively,

$$
\begin{aligned}
L^{\prime}[u]_{C} & =\frac{1}{h^{2}}\left(u_{j_{1}+1, j_{2}}+\frac{1}{2}\left(u_{j_{1}, j_{2}+1}+u_{j_{1}, j_{2}-1}\right)+\left(\frac{(\mathcal{K} h)^{2}}{2}-2\right) u_{j_{1}, j_{2}}\right) \\
& =-\frac{1}{h} \frac{\partial u}{\partial n} .
\end{aligned}
$$

The right hand side of equation (4.12) represents the imposed normal derivative. In each node $m \in \partial \Omega^{\prime}$, the normal vector $\mathbf{n}=\left(n_{j_{1}}, n_{j_{2}}\right)$ can be obtained by assembly of the values in Table 4 . These values are the same for FD and FEM. In the case of node C, elements $q_{1}$ and $q_{4}$ should be considered, obtaining $n_{j_{1}}=-1 / h$ and $n_{j_{2}}=0$.

TABLE 4. Two-dimensional discrete approximation of normal vector in brick-shaped boundaries (pointing outward to the fluid domain).

\begin{tabular}{ccc} 
Existing square & $n_{j_{1}}$ & $n_{j_{2}}$ \\
\hline$q_{1}$ & $-1 / 2 h$ & $-1 / 2 h$ \\
$q_{2}$ & $1 / 2 h$ & $-1 / 2 h$ \\
$q_{3}$ & $1 / 2 h$ & $1 / 2 h$ \\
$q_{4}$ & $-1 / 2 h$ & $1 / 2 h$ \\
\hline
\end{tabular}


It is now possible to consider some boundary conditions. In the Neumann case, the value of $\frac{\partial u}{\partial n}=\mathbf{n} \cdot \nabla u$ is directly considered in equation (4.12) (null value for a rigid scatterer). This modifies the force vector for Robin boundaries $\frac{\partial u}{\partial n}=-A_{R} u$, where $A_{R}$ is a constant. This affects the coefficient of $u_{j_{1}, j_{2}}$ in the diagonal of the system matrix. Dirichlet boundary conditions can be considered [18].

In the case of an FEM-based operator, Neumann and Robin boundary conditions are imposed by means of the boundary integral in equation (4.4).

4.3. Discrete Green's functions. The first step of the method is finding the Green's function $G_{j}^{m}$ of $L$.

4.3.1. One-dimensional discrete Green's functions. As the simplest example we consider below a 1D problem, i.e., scattering by an obstacle on a line. Of course, this example is of no practical interest; however, this example can be used to demonstrate how the CFIE approach removes the spurious resonances.

The mesh $\Omega$ is the whole line, and matrix $\beta$ is given by the relation:

$$
\beta_{j, q}= \begin{cases}h^{-2}, & q=j \pm 1 \\ -2 h^{-2}+\mathcal{K}^{2}, & q=j \\ 0, & \text { otherwise }\end{cases}
$$

The analytical expression for the Green's function is obvious:

$$
G_{j}^{m}=A \exp i \kappa|j-m|,
$$

where $\kappa$ is the discrete wavenumber, $A$ is a constant parameter and $i=\sqrt{-1}$. Wavenumber $\kappa$ is as follows:

$$
\kappa=2 \arcsin (\mathcal{K} h / 2)
$$

Finally, using that for $j=m, L\left[G^{m}\right]_{j}=1$ the expression of $A$ is found as

$$
A=\frac{1}{2(\exp \{i \kappa\}-1) h^{-2}+\mathcal{K}^{2}} .
$$


4.3.2. Two-dimensional discrete Green's functions. Assume that $\beta_{j, q}$ is given by subsection 4.1 . Construct $G_{j}^{m}$ using the Fourier transform. Let the source node $m$ coincide with the origin $m=0$ (all other cases can be obtained by translating the Green's function along the coordinate lines). Introduce the values $j_{1}$ and $j_{2}$ as integer coordinates of node $j$. Represent the Green's function in the form:

$$
G_{j}^{0}=\frac{1}{(2 \pi)^{2}} \iint_{-\pi}^{\pi} A\left(\xi_{1}, \xi_{2}\right) \exp \left\{-i\left(\xi_{1} j_{1}+\xi_{2} j_{2}\right)\right\} d \xi_{1} d \xi_{2} .
$$

The inverse transform is as follows:

$$
A\left(\xi_{1}, \xi_{2}\right)=\sum_{j_{1}=-\infty}^{\infty} \sum_{j_{2}=-\infty}^{\infty} G_{j}^{0} \exp \left\{i\left(\xi_{1} j_{1}+\xi_{2} j_{2}\right)\right\} .
$$

Apply operator $L$ to (4.17):

$$
\begin{aligned}
\delta_{j_{1}, 0} \delta_{j_{2}, 0} & =\frac{1}{h^{2}(2 \pi)^{2}} \iint_{-\pi}^{\pi} \sigma\left(\xi_{1}, \xi_{2}, k\right) A\left(\xi_{1}, \xi_{2}\right) \\
& \times \exp \left\{-i\left(\xi_{1} j_{1}+\xi_{2} j_{2}\right)\right\} d \xi_{1} d \xi_{2}
\end{aligned}
$$

Applying the inversion formula to (4.19), it is concluded that the two-dimensional Green's function can be expressed as:

$$
G_{j}^{0}=\frac{h^{2}}{(2 \pi)^{2}} \iint_{-\pi}^{\pi} \frac{\exp \left\{-i\left(\xi_{1} j_{1}+\xi_{2} j_{2}\right)\right\}}{\sigma\left(\xi_{1}, \xi_{2}\right)} d \xi_{1} d \xi_{2}
$$

Analytical integration on the variable $\xi_{2}$ of equation (4.20) is done. The procedure is similar to the one proposed in [15] and references therein. This leads to one variable integral for the two-dimensional Green's function that is done numerically in the complex plane. A proper integration path that turns around the singular points or poles is chosen. This path is as close as needed to the singular points. This is based on the limit absorption principle (making the limit for the imaginary part of $\mathcal{K}$ tend to 0 ). Consequently, Green's functions calculated by means of this procedure satisfy the Sommerfeld radiation condition.

For the operators defined here in subsection 4.1, $\sigma$ can be expressed 
as $\sigma\left(\xi_{1}, \xi_{2}\right)=A+B \cos (\Xi)$ and equation (4.20) leads to:

$$
G_{j}^{0}=\frac{h^{2}}{2 \pi} \int_{-\pi}^{\pi} \frac{\exp \left\{-i\left(\xi_{1} j_{1}+\Xi j_{2}\right)\right\}}{i B \sin (\Xi)} d \xi_{1}
$$

For the 'FD'case, we have

$$
\Xi\left(\xi_{1}\right)=\arccos \left(2-\cos \xi_{1}-h^{2} \mathcal{K}^{2} / 2\right) \text { and } B=2 .
$$

Its analogue for the 'FEM' operator is

$$
B=2\left[2\left(\frac{(\mathcal{K} h)^{2}}{36}+\frac{1}{3}\right) \cos \left(\xi_{1}\right)+\left(\frac{(\mathcal{K} h)^{2}}{9}+\frac{1}{3}\right)\right]
$$

and

$$
\Xi\left(\xi_{1}\right)=\pi-\arccos \left(\frac{\left(2(\mathcal{K} h)^{2}+6\right) \cos \left(\xi_{1}\right)+4(\mathcal{K} h)^{2}-24}{\left((\mathcal{K} h)^{2}+12\right) \cos \left(\xi_{1}\right)+2(\mathcal{K} h)^{2}+6}\right) .
$$

Equations 4.23 and 4.24 can be simplified if the mass is lumped leading to

$$
\begin{aligned}
B & =\frac{4 \cos \left(\xi_{1}\right)+2}{3} \\
\Xi\left(\xi_{1}\right) & =\pi-\arccos \left(\frac{2 \cos \left(\xi_{1}\right)+3(\mathcal{K} h)^{2}-8}{4 \cos \left(\xi_{1}\right)+2}\right) .
\end{aligned}
$$

An alternative formulation for the ' $F D$ ' case [29] where no analytical integration is done on $\xi_{2}$ leads to:

$$
G_{j}^{0}=\frac{h^{2}}{\pi^{2}} \int_{0}^{\pi} \int_{0}^{\pi} \frac{\cos \left(j_{1} \xi_{1}\right) \cos \left(j_{2} \xi_{2}\right)}{(\mathcal{K} h)^{2}-4+2 \cos \left(\xi_{1}\right)+2 \cos \left(\xi_{2}\right)} d \xi_{1} d \xi_{2} .
$$

Equation (4.27) is more complicated than equation (4.21). However, an analytical solution based on hypergeometric functions exists [24]. An alternative is proposed [33] where the integral is calculated by means of recurrence formulas. Unfortunately, numerical errors due to the recurrence are large for the usual values of $j_{1}$ and $j_{2}$ required by realistic examples and the general purposes of the method presented $[5]$.

A very important contribution of [29] are the simplified formulas that successfully approximate equation (4.27) for large values of $j_{1}$ and $j_{2}$. There is an equivalent three-dimensional version [9]. Without 
them, the implementation of the method proposed here would be more difficult and probably computational costs would be higher.

The option chosen for the examples shown here is to perform a numerical integration of equation (4.21) for the lower values of $j_{1}$ and $j_{2}$ and use asymptotic formulas [29] when possible.

Figure 5 illustrates the dependence between the discrete Green's function precision and how many intervals are used with piecewise constant integration along the integration path. The source is centered at the origin, and the results are for nodes along the $j_{1}$ axis. The error for other nodes like the ones in the diagonal $j_{1}=j_{2}$ is smaller. The plots are very similar for the FEM-based operator.

Several 'rules of thumb' can be obtained from Figure 5: i) The number of integration intervals must always be larger than the maximum value of $j_{1}$ required; ii) it is better not to use asymptotic formulas for $j_{1}<20$.

The results shown in Figure 5 (b) illustrate how important it is to tabulate the values of the discrete Green's function and avoid repetitive calculation of them.

In a problem with $N$ nodes on the scatterer boundary, the number of operations (additions and multiplications) required in order to solve the dense linear systems of equations is $o\left(2 N^{3} / 3\right)$. Moreover, it is required to perform $o\left(2 \alpha N^{2}\right)$ operations and $o\left(\alpha N^{2}\right)$ evaluations of the discrete Green's function in order to compute the coefficients of the system matrix. Here $\alpha$ represents the number of nodes of the truncated operator. For the node $A$ in Figure 2 (c), $\alpha=4$ in the FD-based operator and $\alpha=6$ in the FEM-based operator. But, in any of the cases, $\alpha \ll N$. However, the number of operations required to evaluate the discrete Green's function is comparable or larger than $N$. As shown in Figure 5, the singularity in the integral in equation (4.21) may lead to the use of quite a large number of integration points. In addition, the integrand contains trigonometric functions whose repetitive evaluation tends to be expensive. This can cause the computation of the matrix coefficients to be slower than the solution of the linear system. The system matrices for the examples shown in the following sections are calculated around 100 times faster if discrete Green's function values are precomputed. It is good to verify that most of the calculation time is due to the solver routine. 


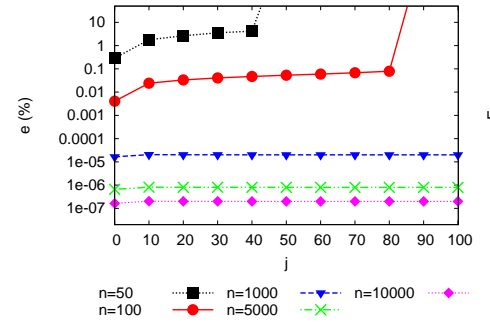

(a)

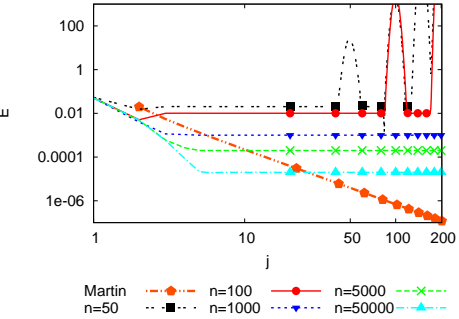

(b)

FIGURE 5. Numerical error in the evaluation of the discrete Green's function along the $j_{1}$ axis $\left(j_{2}=0\right)$ for the finite difference based operator. $n$ is the number of intervals used in piecewise constant integration and the dimensionless wavenumber is $k=0.9$ : (a) relative difference with a reference value obtained by means of 200000 intervals; (b) absolute error in the evaluation of the operator $E=\left|L\left[G^{0}\right]_{j_{1}}-\delta_{0, j_{1}}\right|$. 'Martin' is the asymptotic formula proposed in [29].

4.4. Computational aspects. In terms of memory storage and calculation times required to solve linear systems of equations, the computational costs of the proposed method are comparable with those of the BEM. Both methods use a full system matrix which is the element consuming more memory. The linear systems of equations can be solved by means of direct solvers if they are small but, in general, an iterative solver is required (even if the system matrix can be stored, the direct solver is too slow).

To reduce the condition number of matrices is needed in order to use iterative solvers. In that sense, the integral formulation is relevant [11], and CFIE becomes very important. It is shown in the numerical examples how CFIE drastically improves the condition number of the system matrix for all wavenumbers.

The proposed formulation also avoids boundary integrals. This means that the calculation of the system matrix coefficients can be done in a faster way. This is very relevant in order to extend the technique with the fast multipole methods [47] idea. In these cases, the improvement of the matrix conditioning due to CFIE is very often combined with GMRES (or block GMRES). The whole system matrix 
is never in the memory, and their blocks are generated when required. An important aspect of that methods is the ability to build this block (that implies boundary integrals) very fast.

In addition to these general considerations, some possible guidelines in order to implement a code are:

- The information to be stored is, for each node in the scatterer boundary: the coordinates and the node type. Thus, a function that provides this information for a given geometry is required.

- The unknowns of the linear systems of equations are the nodal values at the boundary. Then, it is important to establish some ordering of them to be assembled. Fast searches along the boundary node list are required. This must be done many times during assembly, pre and post processes.

- The operator $L^{\prime}$ defined in Table 3 can be considered as a function where the input data will be the node coordinates and the node type and as output will provide: coordinates of neighboring nodes and coefficients $\beta^{\prime}$.

- Equation (4.21) is a non-trivial oscillatory integral that must be done for each required value of $G_{j}^{0}$. This is a time consuming operation. It is very important to pre-compute the values of $G_{j}^{0}$ for every wavenumber $\mathcal{K}$ because they are needed several times. The performance of the method highly depends on this step.

5. Numerical examples. The first example is a one-dimensional problem with an available analytical solution. Moreover, the boundary integral formulation is reduced to the solution of a linear system with two equations. This simplicity is helpful for illustrating all the steps of Sections 2 and 3. The other examples are two-dimensional problems where the scatterer is L-shaped, a rectangle or a circle. They are used in order to show numerically some of the properties of the method.

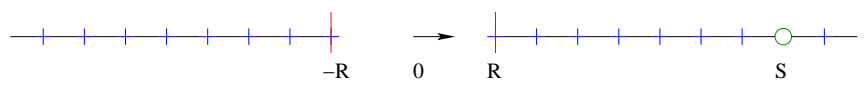

FiguRE 6. One-dimensional scattering problem. Scatterer placed between nodes $-R$ and $R$. Unit point source placed at node $S$. 
5.1. One-dimensional scattering. The one-dimensional problem on Figure 6 is solved. The scattering object is placed between the nodes $-R=-20$ and $R=20$. A point source that generates the wave is placed at the node $S=32$. The rigid boundary of the scatterer is composed by two nodes. In this Neumann boundary, null normal derivative of the total field $u$ is imposed. Then, the problem is reduced to a system of two linear equations where the unknowns are the value of $u$ at these two nodes:

$$
\mathbf{M}\left[\begin{array}{c}
u_{R} \\
u_{-R}
\end{array}\right]=\mathbf{F f}
$$

$\mathbf{M}$ is the system matrix that can be calculated according to equation (3.11):

$$
\mathbf{M}=\left[\begin{array}{cc}
1+b_{R}^{R}+\frac{\nu}{h^{2}}\left(1-\frac{(\mathcal{K} h)^{2}}{2}+b_{R}^{R+1}\right) & b_{-R}^{R}+\frac{\nu}{h^{2}} b_{-R}^{R+1} \\
b_{R}^{-R}+\frac{\nu}{h^{2}} b_{R}^{-R-1} & 1+b_{-R}^{-R}+\frac{\nu}{h^{2}}\left(1-\frac{(\mathcal{K} h)^{2}}{2}+b_{-R}^{-R-1}\right)
\end{array}\right]
$$

Coefficients $b$ are defined in equation (3.5). A more simplified expression of the matrix coefficients can be obtained by means of the onedimensional Green's function described in subsection 4.3 (equations 4.14, 4.15 and 4.16).

$$
\begin{aligned}
\mathbf{M}_{1,2}= & \mathbf{M}_{2,1}=\frac{\exp \{i 2 \kappa R\}}{2}\left(1+\frac{\nu}{h^{2}} \exp \{i \kappa\}\right) \\
\mathbf{M}_{1,1}= & \mathbf{M}_{2,2}=\frac{1}{2}+\frac{\nu}{h^{2}}\left(1-\frac{(\mathcal{K} h)^{2}}{2}+\frac{A}{h^{2}}\right. \\
& \left.\times\left(1-\exp \{i \kappa\}\left(1-\frac{(\mathcal{K} h)^{2}}{2}\right)\right)\right) .
\end{aligned}
$$

The force vector $\mathbf{f}$ is also obtained, thanks to equations 3.6 and 3.8:

$$
\mathbf{F f}=\left[\begin{array}{c}
f_{S} G_{S}^{R}+\frac{\nu}{h^{2}} f_{S} G_{S}^{R+1} \\
f_{S} G_{S}^{-R}+\frac{\nu}{h^{2}} f_{S} G_{S}^{-R-1}
\end{array}\right]
$$

where $f_{S}=1$ is due to the type of source considered and $G$ is the discrete Green's function. Once the boundary values $u_{R}$ and $u_{-R}$ are obtained after the resolution of equation (5.1), the value of $u$ in all the other nodes can be calculated by means of equation (3.6). Considering 
only the non-null coefficients:

$$
u_{m}=f_{S} G_{S}^{m}-u_{R} b_{R}^{m}-u_{-R} b_{-R}^{m} .
$$

The problem has the following exact solution:

$$
u_{j}=\frac{1}{2 i k}(\exp \{i \mathcal{K} h|j-S|\}+\exp \{i \mathcal{K} h(j+S)\}) .
$$

It has been considered as a reference.

Figure 7 (a) shows the inverse of the condition number of matrix $\mathbf{M}$ for several values of the parameter $\nu / h^{2}$. The case $\nu / h^{2}=0$ corresponds to the solution of the problem by means of the boundary integral equation (BIE) equation (3.6). The other cases use equation (3.8); it is a combined field integral equation (CFIE). The eigenfrequencies of the scatterer (with Dirichlet boundary conditions and taking into account the numerical dispersion) are indicated with pink crosses. They can be calculated by means of equation (4.15):

$$
\mathcal{K}_{n}=\frac{2}{h} \sin \left(\frac{1}{2} \kappa_{n}\right)=\frac{2}{h} \sin \left(\frac{n \pi}{4 R}\right) \quad n=1,2, \ldots
$$

The condition number of singular matrices is infinite. Its inverse is 0 just in the eigenfrequencies of the scatterer when $\nu / h^{2}=0$, which are the spurious eigenfrequencies of the problem. This never happens when the combined field formulation is used $(\nu \neq 0)$. In [1], the value of $\nu / h^{2}=i$ is used in the absence of a more detailed analysis. This seems correct in our case and avoids the singularity of matrix $\mathbf{M}$ for all the wavenumbers. Other values have been tested here without obtaining an improvement in the condition number with respect to $\nu / h^{2}=i$. Note that the value of ' $u_{-R}, C F I E$ ' is always null.

The relative error of $u_{R}$ and $u_{-R}$ depending on the dimensionless wavenumber for $\nu / h^{2}=i$ has been plotted in Figure 7 (b). The general trend is the same for BIE and CFIE. $u_{-R}$ has only rounding errors because it is exactly 0 in both cases. $u_{R}$ is quite dependent on the dispersion of the incident wave. However, the important difference between both methods is found at the spurious frequencies of the problem corresponding to the eigenfrequencies of the scatterer (interior problem with Dirichlet boundary conditions). For these wavenumbers, BIE provides bad results for both $u_{R}$ and also $u_{-R}$ while CFIE is still coherent with the exact solution. 


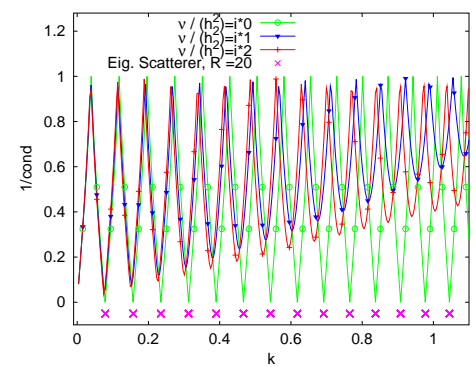

(a)

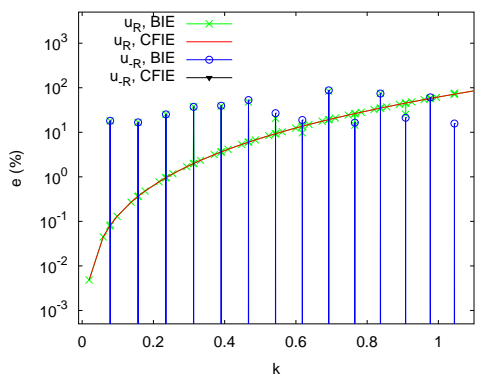

(b)

FIGURE 7. One-dimensional scattering problem: (a) Inverse of the condition number depending on the parameter $\nu / h^{2}$ for each dimensionless wavenumber $k=\mathcal{K} h$, 'Eig.Scatterer' are the eigenfrequencies of the scatterer; (b) Relative error of the values $u_{R}$ and $u_{-R}$ at both sides of the scatterer for the boundary integral formulation (BIE) and the combined field integral equation (CFIE), $\nu / h^{2}=i$.

The field solution for two particular wavenumbers has been plotted in Figure 8. In situation (a), both methods provide a correct solution. The wavenumber is calculated with $n=4.5$ in equation (5.8). On the contrary, in situation (b), only CFIE is close to the exact solution. In that case, $n=4$, which means that we are exactly in one of the spurious resonances of the problem. The wave of the BIE solution in the left domain is, of course, caused by the failure of the formulation at that frequency. It is shown as a 'mirror effect.'

5.2. Scattering by two-dimensional objects. Three different scattering objects have been considered: an 'L,' a rectangle and a circle. The former is more adequate for the proposed technique because its geometry can be exactly approximated. The latter is a case with analytical solution [10]. Moreover, it can be used to see the performance of the method for curved boundary geometries.

5.2.1. Straight edges. The situation of Figure 9 (a) is considered. A point source generates a cylindrical wave that is scattered by a rectangle. The source is placed at the position $\left(X_{S}=0 \mathrm{~m} ; Y_{S}=4 \mathrm{~m}\right)$. 


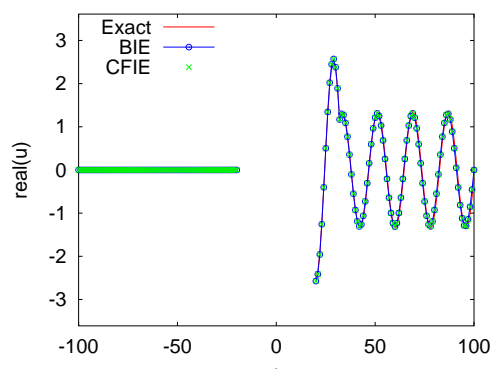

(a)

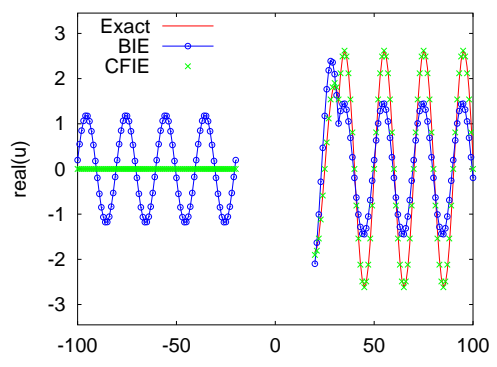

(b)

FIGURE 8. Detail of the solutions for a one-dimensional scattering problem: (a) wavenumber which does not coincide with an eigenfrequency of the scatterer, all methods provide equivalent solutions; (b) wavenumber which coincides with an eigenfrequency of the scatterer, pathological behavior of the BIE solution seen as a 'mirror effect.'

$\otimes(\mathrm{Xs}, \mathrm{Ys})$

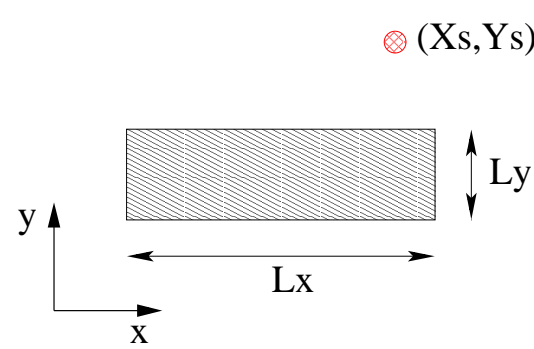

(a)

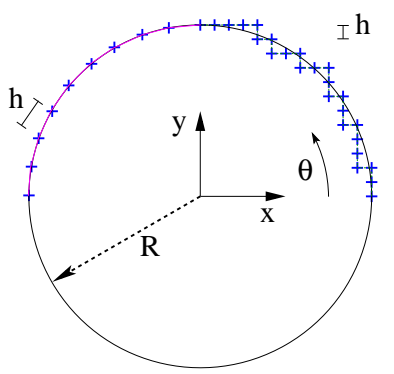

(b)

FIGURE 9. Sketches and notation of the two-dimensional examples. Scattering of cylindrical incident wave by: (a) a rectangle; (b) a circle. The brick discretization of the circle is plotted for $\theta$ varying between 0 and $\pi / 2 \mathrm{rad}$. The BEM discretization is plotted for $\theta$ varying between $\pi / 2 \mathrm{rad}$ and $\pi \mathrm{rad}$.

The rectangle has dimensions $\left(L_{X}=2.0 \mathrm{~m} ; L_{Y}=1.6 \mathrm{~m}\right)$ and the bottom left corner is placed at the position $(-1.6 \mathrm{~m} ; 0 \mathrm{~m})$. 
In a first example, the incident field is considered by means of equation (3.9) with

$$
u^{\mathrm{inc}}(r)=\frac{-i}{4} H_{0}^{(1)}(\mathcal{K} r) .
$$

Four different methods are compared:

(i) The boundary integral of equation (3.6) (BIE);

(ii) The boundary integral of equation (3.8) with a coefficient $\nu=1$ $(\mathrm{BIE}+\mathrm{CFIE})$

(iii) A standard boundary element solution based on Green's formula. The variables are interpolated by means of piecewise constant elements with the collocation point in the middle of the element (BEM);

(iv) The same boundary element formulation but taking into account the CFIE [8] (BEM + CFIE).

Due to the lack of analytical solutions for this example, the reference solution is taken as the BEM + CFIE option, but dividing the element size by two. A frequency analysis is performed. The analyzed dimensionless wavenumbers $(k=\mathcal{K} h)$ are chosen as: eigenfrequencies of the rectangular shape by taking into account the 'exact' wavenumber, eigenfrequencies of the rectangular shape by taking into account the dispersion in the numerical wavenumber (in the same way as equation (5.8) but in two directions), some frequencies around these values and frequencies distributed in a uniform way along the spectrum. The frequency range has been limited to values of dimensionless wavenumber below 1. For this value, there are approximately six nodes per wave length. The relative error is calculated by means of the energy norm on the boundary of the scattering object:

$$
e=\frac{\int_{\partial \Omega^{\prime}}\left|u-u_{\mathrm{ref}}\right|^{2} d S}{\int_{\partial \Omega^{\prime}}\left|u_{\mathrm{ref}}\right|^{2} d S} .
$$

The results for a grid size $h=0.1 \mathrm{~m}$ are shown in Figure 10. The first thing to be noted is that both the BIE and the BEM suffer from large errors at (and around) the eigenfrequencies of the rectangle (with Dirichlet boundary conditions) as shown in Figure 10 (a). The dimensions of the rectangle as well as the grid size $h$ have been chosen in order to show it clearly. However, for larger rectangles and higher frequencies (smaller values of $h$ ), the effect of the spurious 
frequencies is much more dramatic because of the larger modal density of the rectangle. This numerical pathology is overcome when a CFIE formulation is used. The success of the technique is valid in all cases, as well as for the discrete boundary integral equation proposed here. CFIE formulation improves the conditioning of the system matrix. The 2-norm condition number of the system matrix is plotted in Figure 10 (b). We can see how CFIE keeps the condition number in all cases below 50. On the contrary, without CFIE, large values of the condition number are found around the spurious frequencies.

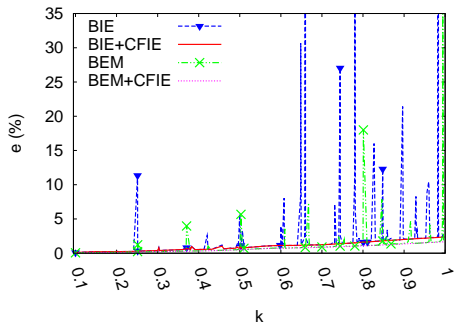

(a)

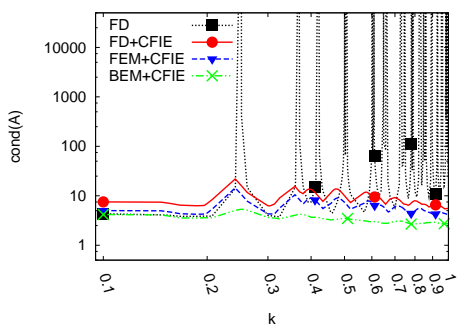

(b)

FiguRE 10. Effect of CFIE: (a) Relative error around a rectangular scatterer (incident field according to $(5.9), h=0.1 \mathrm{~m}$ ); (b) 2-norm condition number of the system matrix depending on the wavenumber. Among all cases without CFIE only FD-based operator is plot for clarity.

The behavior of the numerical models around spurious frequencies is shown in Figure 11 and, for the sake of clarity, some meaningful values are listed in Table 5. The behavior of the BEM and the boundary algebraic equations is quite different. In the BEM, the effect of the spurious frequency on the solution can be noted in a reasonably large bandwidth $(\Delta k \simeq 0.05 \mathrm{~Hz}$ in Figure $11(\mathrm{~b}))$. However, this is not the case in boundary algebraic equations where this bandwidth is extremely narrow $\left(\Delta k \simeq 10^{-9} \mathrm{~Hz}\right.$ from the values in Table 5). Another difference is that the BEM seems more sensitive to the ill-conditioning of matrices. The numerical error increases for values of the system condition matrix around $10^{2}$. On the contrary, in boundary algebraic equations, this condition number must be larger than $10^{8}$ in order to see its affect on the quality of the solution. 


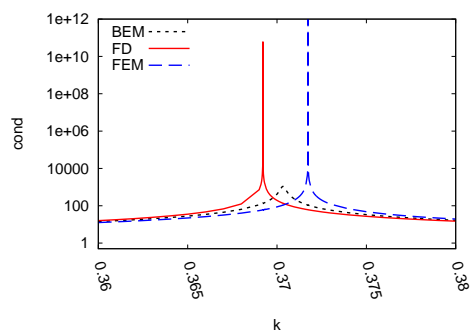

(a)

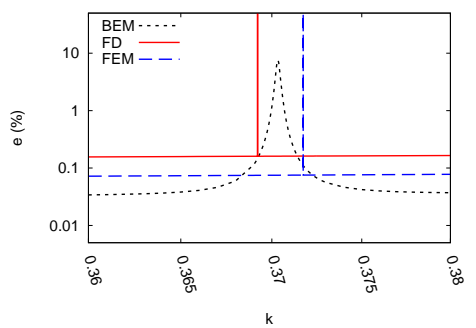

(b)

FIGURE 11. Detailed effect of a spurious frequency in BEM and for the FD and FEM based operator: (a) 2-norm condition number of system matrices without CFIE; (b) relative error.

TABLE 5. Meaningful numerical values of Figure 11.

\begin{tabular}{cccc}
\hline $\mathrm{k}$ & $\begin{array}{c}\text { FD-based } \\
\text { cond }\end{array}$ & cond (CFIE) & $\mathrm{e}(\%)$ \\
0.369200000000000 & $1.45 \times 10^{4}$ & 12.4 & 0.16 \\
0.369209000000000 & $1.00 \times 10^{5}$ & 12.4 & 0.16 \\
0.369210515000000 & $5.02 \times 10^{7}$ & 12.4 & 0.16 \\
0.369210518005500 & $5.40 \times 10^{9}$ & 12.4 & 1.26 \\
0.369210518033600 & $6.19 \times 10^{10}$ & 12.4 & 141.7 \\
\hline & FEM-based & & \\
$\mathrm{k}$ & cond & cond (CFIE) & $\mathrm{e}(\%)$ \\
0.371730000000000 & $2.59 \times 10^{4}$ & 8.07 & $7.53 \times 10^{-2}$ \\
0.371735700000000 & $7.03 \times 10^{5}$ & 8.07 & $7.53 \times 10^{-2}$ \\
0.371735900000000 & $8.60 \times 10^{6}$ & 8.07 & $7.53 \times 10^{-2}$ \\
0.371735917801198 & $1.03 \times 10^{12}$ & 8.07 & 148 \\
\hline & BEM & & \\
$\mathrm{k}$ & cond & cond $(\mathrm{CFIE})$ & $\mathrm{e}(\%)$ \\
0.360000000000000 & $1.43 \times 10^{1}$ & 4.06 & $3.40 \times 10^{-2}$ \\
0.369000000000000 & $1.13 \times 10^{2}$ & 4.22 & $1.14 \times 10^{-1}$ \\
0.370220000000000 & $8.48 \times 10^{2}$ & 4.23 & 4.63 \\
0.370331500000000 & $1.08 \times 10^{3}$ & 4.23 & 7.56 \\
\hline
\end{tabular}

The precision of the method for several operators is shown in Figure 12. In addition to the variations of the original operator described in subsection 4.1, the direct truncation of the finite difference operator is considered (FD trunc). This is the simplest case where all grid nodes 
on the scatterer are removed (the weight of the central node is adapted taking into account the number of adjacent nodes). The relative error of a BEM solution using piecewise constant elements of size $h$ is also included in the comparison.

Two scatterers are considered: rectangular (the same as before) and L-shaped. The bottom side of the L-shaped scatterer is horizontal and coincides with the bottom side of the rectangle. Both scatterers are $2.0 \mathrm{~m}$ width. The $\mathrm{L}$ is $3.2 \mathrm{~m}$ height and $0.4 \mathrm{~m}$ thick, with the source of the incident wave placed at $\left(X_{S}=0 \mathrm{~m} ; Y_{S}=4 \mathrm{~m}\right)$.

It can be seen how, as expected, due to the simplification in the geometry description, the method proposed here is a bit less accurate than the BEM. This difference in accuracy is smaller for the L-shaped scatterer. It could mean that, when the solution has stronger singularities, the difference (in terms of precision) between the proposed method and the BEM is reduced. However, the convergence order is the same in both methods. The slope of that curves is reduced when the scatterer has a non-soft shape (i.e., sharp contours or corners) [22]. This leads to solutions including strong singularities. The slope is around 2 (1 in $L_{2}$ norm), and it could be around 4 (2 in $L_{2}$ norm) in a case without singularities. Errors are larger in the case of the L-shaped scatterer (for all the operators and in both methods) because the solution of the problem has stronger singularities due to the re-entrant corner.

In both scatterers, the direct truncation of the operator (FD trunc) leads to larger errors. A proper truncation of the operator $L$ that takes into account the boundary conditions improves the precision of the solution. The main difference between the options ' $F D$ ' and 'FD trunc' is that the first one represents a second order approximation of the normal derivative around the scatterer while the second one does not.

In the rectangular scatterer, the errors are smaller if the operator $L$ is based on a finite element (FEM, FEM lumped) instead of finite differences (FD). However, it is not the case for the L-shaped scatterer. The difference between the options 'FEM' and 'FEM lumped' is very small. Consequently, it is better to use an operator based on finite elements with a lumped matrix because the expressions to evaluate the discrete Green's functions in subsection 4.3 are much simpler (this also reduces computation time). 


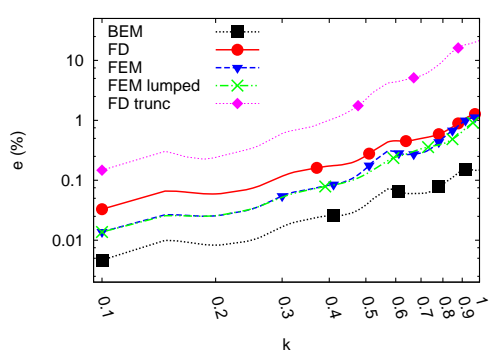

(a)

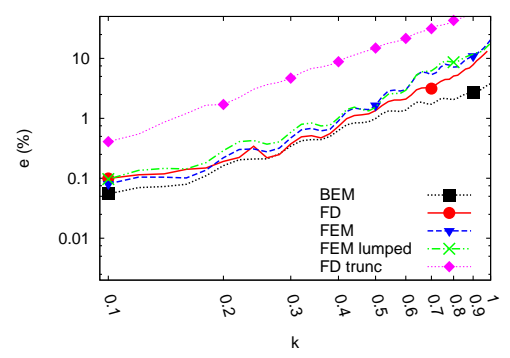

(b)

FIGURE 12. Relative error around a scatterer: (a) rectangular; (b) Lshaped. Comparison between several operators based on: finite differences (FD), finite element (FEM), finite element with lumped mas matrix (FEM lumped) and finite differences with direct truncations (FD trunc). A BEM solution using piecewise constant elements is also included. All the cases use CFIE.

The method has also been compared with a finite difference analysis in the time-domain (FDTD). The wave equation is solved in the timedomain. A wave with compact support is generated in the source position and propagated in an unbounded domain until introduction of the scatterer. The outputs from the FDTD analysis are the timehistories of the source force and the variable around the scatterer. They can be transformed into the frequency domain by means of a discrete Fourier transform (DFT). The ratio between the Fourier transform of the received signal divided by the Fourier transform of the source force has to be equivalent to a frequency domain analysis using equation (1.1) where the force term is a Dirac delta placed in the source position. In the FDTD analysis, the incident and reflected waves have numerical dispersion. Thus, in order to make a fair comparison, the incident field is generated by a Dirac delta force vector in the frequency-domain analysis placed at the position $\left(s_{1}, s_{2}\right)$

$$
f_{j}=\delta_{j_{1}, s_{1}} \delta_{j_{2}, s_{2}},
$$

instead of the dispersion-free incident field of equation (5.9) in equation (3.9). The use of a discrete Green's function ensures that the incident field also has numerical dispersion. 
A comparison of the time and frequency analyses is shown in Figure 13. The reference solution is again the BEM + CFIE formulation with a finer mesh. The wavenumbers analyzed in the 'BIE' and 'BIE+CFIE' options are chosen as before. The wavenumbers obtained by the discrete Fourier transform coincide with the symbol (dark square). Two aspects must be taken into account in order to fix the validity range of the DFT output. On one hand, only the solutions with wavenumbers with a significant contribution in the excitation have sense. This frequency range can be increased by making the impulse sharper (with shorter time duration) and closer to a Dirac delta. On the other hand, the grid size indirectly determines the maximum wavenumber with physical meaning. It does not seem adequate to consider frequencies with less than six elements per wave length. For this reason, only dimensionless wavenumber values in the range $(0 ; 1)$ have been considered.

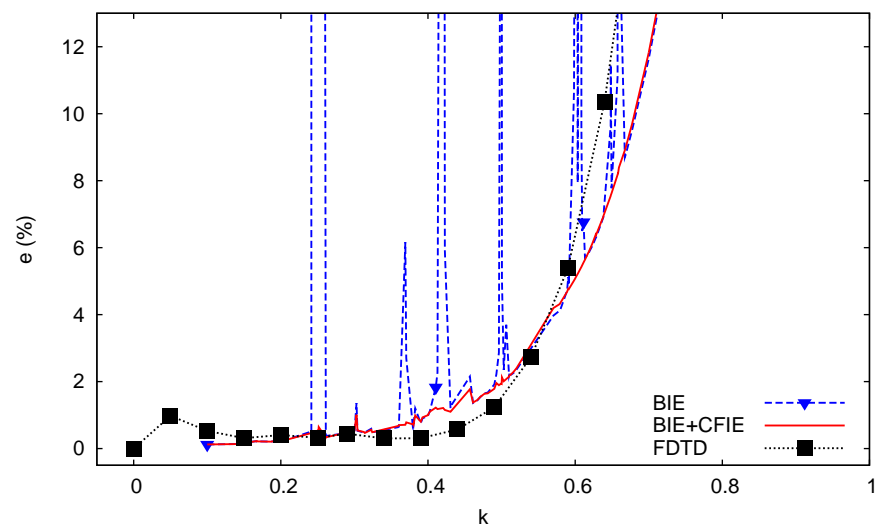

FIGURE 13. Relative error around a rectangular scatterer (the incident field has numerical dispersion, $h=0.1 \mathrm{~m}$ ). Comparison between methods: the proposed BIE provides similar results than a finite difference time-domain analysis (post-processed in frequencies).

The relative error has similar values and follows the same trend in both the 'BIE+CFIE' and the frequency post-processed 'FDTD.' In both cases, it drastically increases after $k=0.5$. It has to be noted that the BEM does not introduce dispersion in the incident field because its 
analytical expression is included directly in the force term. Thus, we are comparing two solutions where the incident field has numerical dispersion with a reference solution with dispersion free incident field. When analyzed in detail, it can be seen how the numerical error is mainly caused by a phase difference in the waves but their module is similar.

To obtain similar results working in the time-domain and in the frequency-domain is important in order to show that all of the aspects more related to the frequency domain such as discrete Green's functions, the BIE of equation (3.6) or the definition of parameters $b$ in equation (3.5) are properly formulated and implemented.

5.2.2. Curved boundary: circle. Once the properties of the method have been shown in the previous example, a new ingredient is added here: approximation of curved boundaries by means of a brick-shaped geometry. To do that, the scattering of a cylindrical wave caused by a circle is considered. The approximation to curved surfaces by means of stair-stepped discretizations represents an additional source of numerical errors. Some attempts have been made in order to improve this aspect of finite differences in time-domain [43] and in frequency domain $[\mathbf{5 0}, \mathbf{7}]$.

The sketch of the problem can be seen in Figure 8 (b). The radius of the circle is $R=3 \mathrm{~m}$ and the position of the source $\left(X_{S}=\right.$ $\left.0 \mathrm{~m} ; Y_{S}=8 \mathrm{~m}\right)$. The incident field is modeled using equation (5.9) in equation (3.9). All the considerations done for the previous example remain valid with the difference that, now, the reference solution is the series solution [10]. The BEM options use nodes exactly over the radius of the circle (thus, the collocation point in the middle of the element is slightly displaced from the circumference). The reference solution is evaluated on the circle at the same angle $\theta$ as the collocation point. For the BIE options, the nodes of the grid are not exactly over the circle. The reference solution is evaluated over the circle and at the same angle $\theta$ aligned with the grid node.

The results are shown in Figure 14. Again, the use of CFIE dampens the spurious frequencies. It can be seen how this circular example is very favorable for the BEM due to the radial nature of the fundamental solution of the problem. However, the convergence rate is quite similar in both cases. It is around 4 (2 in $L_{2}$ norm) and much larger than in 
Figure 12. This is expected due to the lack of corners in the scatterer geometry which avoids singularities in the solution. Thus, the errors are smaller than for the BIE where the Green function is constructed following the orthogonal directions of the grid. The errors drastically increase after $k=0.5$ for BIE and $k=0.9$ for the BEM.

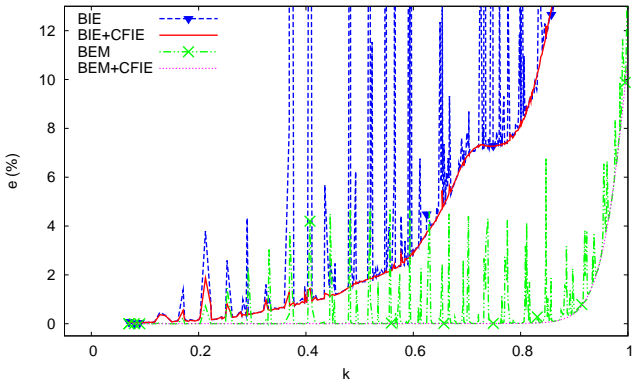

(a)

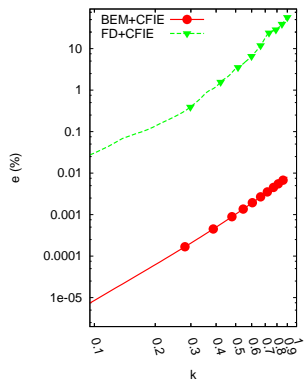

(b)

FIGURE 14. Relative error around a circle-shaped scatterer, comparison between methods: (a) incident field according to (5.9), $h=0.1 \mathrm{~m}$, the standard version of the BEM and the proposed BIE provide spurious solutions in the eigenfrequencies of the scatterer. This problem is overcome with the CFIE versions in both methods; (b) the incident field is a plane wave, accuracy comparison when CFIE is used.

The relative error of Figure 14 is calculated on the boundary of the scattering object $\left(\partial \Omega^{\prime}\right)$. It has two main causes in the proposed method. On one hand, the stair-stepped approximation of the geometry is less important in the evaluation of the solution outside the boundary (far field). On the other hand, the fact remains that the nodes of the grid cannot be exactly placed on the circle. This is improved in the evaluation of the field in other positions (not on the scatterer boundary) by simply interpolating the solution.

6. Conclusions and future developments. A numerical technique for the scattering problems governed by the Helmholtz equation has been presented. It is based on the discrete form of the problem and uses discrete Green's functions. The main properties of the method are: 
- Keeps the properties of the BEM and other techniques based on boundary integral equations in the sense that automatically satisfies the boundary conditions at infinity. This avoids the use of specific techniques such as the perfectly matching layers, the Dirichlet-to-Neumann maps or infinite elements and represents a good option to model unbounded domains in a finite difference based model.

- The discrete equivalent version of the combined field integral equation formulation (CFIE) has been used in order to damp the spurious resonances of the scatterer with success.

- No boundary integral has to be done. This is a very important advantage with respect to other boundary techniques such as the BEM. One of the main drawbacks of the BEM is the need to deal with singular integrals. This problem is even more important when CFIE formulations are used because they require normal derivatives of the original equations which implies an increase on the degree of singularity of the integrals.

- According to the numerical results presented in Figures 12 and 14 (b), the convergence of the method is similar to those of the BEM. It is of order $2\left(L_{2}\right.$ norm $)$ in problems without singularities in the solution and scatterers without corners but the order of convergence is reduced otherwise.

- The idea presented here can be adapted to deal with other equations and problems with minor changes. Two ingredients are required: modify the operator $L^{\prime}$ and use proper discrete Green's functions for the new problem. Both of these seem very feasible because finite differences have been used in many other physical problems and some other discrete Green's functions have also been studied.

Good properties of the method have been shown by means of several examples.

The method presented here is a very promising technique for threedimensional applications. The difficulty to deal with singular and hypersingular integrals is much more important for the BEM in 3D than in $2 \mathrm{D}$. In $2 \mathrm{D}$, this problem can be overcome with minor difficulties as shown in the circle example. However, this will not be the case in 3D. Moreover, in 3D, it is even more necessary to use a CFIE formulation due to the highest modal density of the scatterer. This increases the 
number of spurious frequencies and, in practice, it makes useless a standard BIE formulation. In these cases, the property of avoiding surface integrals will be even more relevant.

\section{REFERENCES}

1. S. Amini and P.J. Harris, A comparison between various boundary integral formulations of the exterior acoustic problem, Comp. Meth. Appl. Mech. Eng. 84 (1990), 59-75.

2. A. Anand, J. Ovall and C. Turc, Well-conditioned boundary integral equations for two-dimensional sound-hard scattering problems in domains with corners, J. Int. Equat. Appl. 24 (2012), 321-358.

3. A. Bamberger, J.C. Guillot, and P. Joly, Numerical diffraction by a uniform grid, SIAM J. Numer. Anal. 25 (1988), 753-783.

4. W. Benthien and A. Schenck, Nonexistence and nonuniqueness problems associated with integral equation methods in acoustics, Comp. Struct. 65 (1997), 295-305.

5. M. Berciu, On computing the square lattice Green's function without any integrations, J. Phys. Math. Theor. 42 (2009), 395207.

6. H. S. Bhat and B. Osting, Diffraction on the two-dimensional square lattice, SIAM J. Appl. Math. 70 (2009), 201-210.

7. S. Britt, S. Tsynkov and E. Turkel, Numerical simulation of time-harmonic waves in inhomogeneous media using compact high order schemes, Commun. Comput. Phys. 9 (2011), 520-541.

8. A.J. Burton and G.F. Miller, Application of integral equation methods to numerical solution of some exterior boundary-value problems, Proc. Roy. Soc. Math. Phys. 323 (1971), 201-210.

9. J. Callaway, Theory of scattering in solids, J. Math. Phys. 5 (1964), 783-798.

10. T.J. Cavicchi and W.D. O'brien, Acoustic scattering of an incident cylindrical wave by an infinite circular-cylinder, IEEE Trans. Ultra. Ferro. Freq. Contr. 35 (1988), 78-80.

11. S.N. Chandler-Wilde, I.G. Graham, S. Langdon and M. Lindner, Condition number estimates for combined potential boundary integral operators in acoustic scattering, J. Integral Equat. Appl. 21 (2009), 229-279.

12. J.T. Chen, I.L. Chen and K.H. Chen, Treatment of rank deficiency in acoustics using SVD, J. Comp. Acoust. 14 (2006), 157-183.

13. K.E. Chen and S. Amini, Numerical analysis of boundary integral solution of the Helmholtz equation in domains with non-smooth boundaries, IMA J. Num. Anal. 13 (1993), 43-66.

14. C.C. Chien, H. Rajiyah and S.N. Atluri, An effective method for solving the hyper-singular integral equations in 3-D acoustics, J. Acoust. Soc. Am. 88 (1990), 918-937.

15. J. Cserti, Application of the lattice Green's function for calculating the resistance of an infinite network of resistors, Amer. J. Phys. 68 (2000), 896-906. 
16. K.A. Cunefare, G. Koopmann and K. Brod, A boundary element method for acoustic radiation valid for all wavenumbers, J. Acoust. Soc. Am. 85 (1989), $39-48$.

17. K. Gerdes, A review of infinite element methods for exterior Helmholtz problems, J. Comp. Acoust. 8 (2000), 43-62.

18. A. Gillman and P.G. Martinsson, Fast and accurate numerical methods for solving elliptic difference equations defined on lattices, J. Comput. Phys. 229 (2010), 9026-9041.

19. M.A. Golberg, Numerical solution of integral equations, Math. Concepts Meth. Sci. Eng., Plenum Press, 1990.

20. L.J. Gray and E. Lutz, On the treatment of corners in the boundary element method, J. Comp. Appl. Math. 32 (1990), 369-386.

21. I. Harari and T.J.R. Hughes, A cost comparison of boundary element and finite element methods for problems of time-harmonic acoustics, Comp. Meth. Appl. Mech. Eng. 97 (1992), 77-102.

22. P. Juhl, A note on the convergence of the direct collocation boundary element method, J. Sound Vibr. 212 (1998), 703-719.

23. J.T. Katsikadelis, Boundary elements: Theory and applications, 1, Elsevier, 2002 .

24. S. Katsura and S. Inawashi. Lattice Greens functions for rectangular and square lattices at arbitrary points, J. Math. Phys. 12 (1971), 1622-1630.

25. R.E. Kleinman and G.F. Roach, Boundary integral-equations for 3dimensional Helmholtz equation, SIAM Rev. 16 (1974), 214-236.

26. G. Kukaba, The boundary element method: Errors and gridding for problems with hot spots, Ph.D. thesis, Eindhoven University of Technology, 2011.

27. S. Liapis, A review of error estimation and adaptivity in the boundary element method, Eng. Anal. Bound. Elem. 14 (1994), 315-323.

28. H. Lim, S.V. Utyuzhnikov, Y.W. Lam, A. Turan, M.R. Avis, V.S. Ryaben'kii, and S.V. Tsynkov, Experimental validation of the active noise control methodology based on difference potentials, AIAA J. 47 (2009), 874-884.

29. P.A. Martin, Discrete scattering theory: Green's function for a square lattice, Wave Motion 43 (2006), 619-629.

30. P.G. Martinsson and G.J. Rodin, Boundary algebraic equations for lattice problems, Proc. Roy. Soc. Math. Phys. Eng. Sci. 465 (2009), 2489-2503.

31. M. Medvinsky, S. Tsynkov, and E. Turkel, The method of difference potentials for the Helmholtz equation using compact high order schemes, J. Sci. Comput. 53 (2012), 150-193.

32. , High order numerical simulation of the transmission and scattering of waves using the method of difference potentials, J. Comput. Phys. 243 (2013), 305-322.

33. T. Morita, Useful procedure for computing lattice Greens function-Square, tetragonal, and Bcc lattices, J. Math. Phys. 12 (1971), 1744-1747. 
34. M. Nabavi, M.H.K. Siddiqui and J. Dargahi, A new 9-point sixth-order accurate compact finite-difference method for the Helmholtz equation, J. Sound Vibr. 307 (2007), 972-982.

35. Y. Okada, A. Shudo, S. Tasaki and T. Harayama, On the boundary element method for billiards with corners, J. Phys., A-Math. Gen. 38 (2005), 6675-6688.

36. B. Ozgener and H.A. Ozgener, Gaussian quadratures for singular integrals in BEM with applications to the 2D modified Helmholtz equation, Eng. Anal. Bound. Elem. 24 (2000), 259-269.

37. O.I. Panich, On the solubility of exterior boundary-value problems for the wave equation and for a system of Maxwell's equations, Uspek. Mat. Nauk. 20 (1965), 221-226.

38. J. Poblet-Puig, V.Yu. Valyaev, and A.V. Shanin, Boundary element method based on preliminary discretization, Math. Mod. Comp. Simu. 6 (2014), 172-182.

39. P.S. Ramesh and M.H. Lean, Accurate integration of singular kernels in boundary integral formulations for Helmholtz equation, Int. J. Num. Meth. Eng. 31 (1991), 1055-1068.

40. P.A. Raviart and J.M. Thomas, Introduction à l'analyse numérique des équations aux dérivées partielles, Collect. Math. Appl. Matr., Masson, 1983.

41. R.J. Riddell, Boundary-distribution solution of the Helmholtz equation for a region with corners, J. Comp. Phys. 31 (1979), 21-41.

42. Dan Rosen and Donald E. Cormack, On corner analysis in the BEM by the continuation approach, Eng. Anal. Bound. Elem. 16 (1995), 53-63.

43. J.A. Russer, P.S. Sumant and A.C. Cangellaris, Modeling of curved boundaries in the finite-difference time-domain method using a lagrangian approach, in Time domain methods in electrodynamics, Springer Proc. Phys. 121 (2008), 55-67, Springer, Berlin.

44. V.S. Ryaben'kii, Difference potentials method and its applications, Math. Nachr. 177 (1996), 251-264.

45. $\quad$ On the method of difference potentials, J. Sci. Comp. 28 (2006), $467-478$.

46. H.A. Schenck, Improved integral formulation for acoustic radiation problems, J. Acoust. Soc. Amer. 44 (1968), 41-58.

47. L. Shen and Y.J. Liu, An adaptive fast multipole boundary element method for three-dimensional acoustic wave problems based on the Burton-Miller formulation, Comp. Mech. 40 (2007), 461-472.

48. I. Singer and E. Turkel, High-order finite difference methods for the Helmholtz equation, Comp. Meth. Appl. Mech. Eng. 163 (1998), 343-358.

49. V. Sladek and J. Sladek, Singular integrals and boundary elements, Comp. Meth. Appl. Mech. Eng. 157 (1998), 251-266.

50. I. Tsukerman, A class of difference schemes with flexible local approximation, J. Comput. Phys. 211 (2006), 659-699.

51. , A singularity-free boundary equation method for wave scattering, IEEE Trans. Ant. Propag. 59 (2011), 555-562. 
52. S.V. Tsynkov, Numerical solution of problems on unbounded domains, A review, Appl. Numer. Math. 27 (1998), 465-532.

53. , On the definition of surface potentials for finite-difference operators, J. Sci. Comput. 18 (2003), 155-189.

54. Xiaojuen Yuan, D. Borup, J.W. Wiskin, M. Berggren, R. Eidens and S.A. Johnson, Formulation and validation of Berenger's PML absorbing boundary for the FDTD simulation of acoustic scattering, IEEE Trans. Ultra. Ferr. Freq. Contr. 44 (1997), 816-822.

Laboratori de Càlcul Numèric, E.T.S. D'Enginyers de Camins, Canals i Ports de Barcelona, Universitat Politècnica de Catalunya. CorresponDence: UPC, Campus Nord B1, Jordi Girona 1, E-08034 Barcelona, Spain Email address: jordi.poblet@upc.edu

Department of Physics, Acoustics Division, Moscow State University, 119992, Moscow, Leninskie Gory, Russia

Email address: valery-valyaev@yandex.ru

Department of Physics, Acoustics Division, Moscow State University, 119992, Moscow, Leninskie Gory, Russia

Email address: a.v.shanin@gmail.com 